\title{
The primate connection
}

\section{Studies comparing HIV infection and its simian counterpart in different monkey species are filling gaps in knowledge, explains Bijal Trivedi.}

ale African green monkeys are notorious for their striking red penis, bright-blue scrotal area and white abdomen. To AIDS researchers, however, these monkeys and another African species, the sooty mangabey, are known for a less visible trait.

Both are natural hosts of the simian immunodeficiency virus (SIV), which is the primate counterpart and ancestor of HIV. Although infected monkeys have more than 100,000 copies of SIV per millilitre of blood, they do not get sick or develop anything resembling AIDS. By contrast, Asian macaques infected with SIV mimic the trajectory of human HIV infection.

Understanding what allows the African monkeys to coexist with SIV could help develop drugs or vaccines against HIV that ape their response.

"Comparative AIDS research
"It is amazing that [the monkeys] are able to switch off these pathways."
CD4 cells in sooty mangabeys have lower levels of the receptor CCR5, which HIV needs to enter the cell. African green monkeys have fewer surface $\mathrm{CD} 4$ receptors, which are required for HIV infection.

"They've chosen two different ways of removing target cells of the virus," notes Daniel Douek, who leads the Human Immunology Section of the US National Institute of Allergy and Infectious Diseases' Vaccine Research Center. "I really think that is the root of why they don't progress [to AIDS]."

Viral load is not the only factor influencing progression. People with low levels of HIV sometimes show serious inflammation and a slow decline in CD4 cells. Some such cases slowly progress to AIDS, says Steven Deeks, professor of medicine at the University of California, San Francisco.

is essential to figure out what protects us from transmission, and what protects us from pathogenesis," says Guido Silvestri, professor of pathology at Emory University.

SIV infections in the natural host monkeys share similarities with human HIV and macaque SIV infections; however, it is the differences that intrigue researchers. For example, unlike humans and macaques, female natural hosts rarely pass the virus on to their offspring.

In people, there is a general trend: the more virus in the body, the faster the progression to AIDS. This is probably why elite controllers, whose immune systems can whittle down viral loads to barely detectable levels, rarely get AIDS (see page S4). By contrast, sooty mangabeys and African green monkeys with high viral loads remain healthy.

What is more, the natural hosts maintain relatively healthy numbers of CD4 cells white blood cells that orchestrate the immune response and are targets of HIV - in both blood and the mucus lining of the genital tract.

\section{Evasive targets}

One key to the monkeys' resilience might be that they have fewer cells that HIV can infect.
"There are people who think it is only the virus, others think it is only inflammation, and we think it is a little of both," adds Deeks.

When the virus first infects the body, humans, macaques and natural hosts mount a dramatic immune response. In sooty mangabeys and African green monkeys, this reverts to baseline levels after about three months, whereas it remains high in humans and macaques throughout the chronic infection.

"It is amazing that [the natural hosts] are able to switch off these pathways of immune response even when virus replication remains consistently high. And it seems that they benefit from doing this switch off,' says Silvestri.

One potential trigger for chronic immune activation in humans
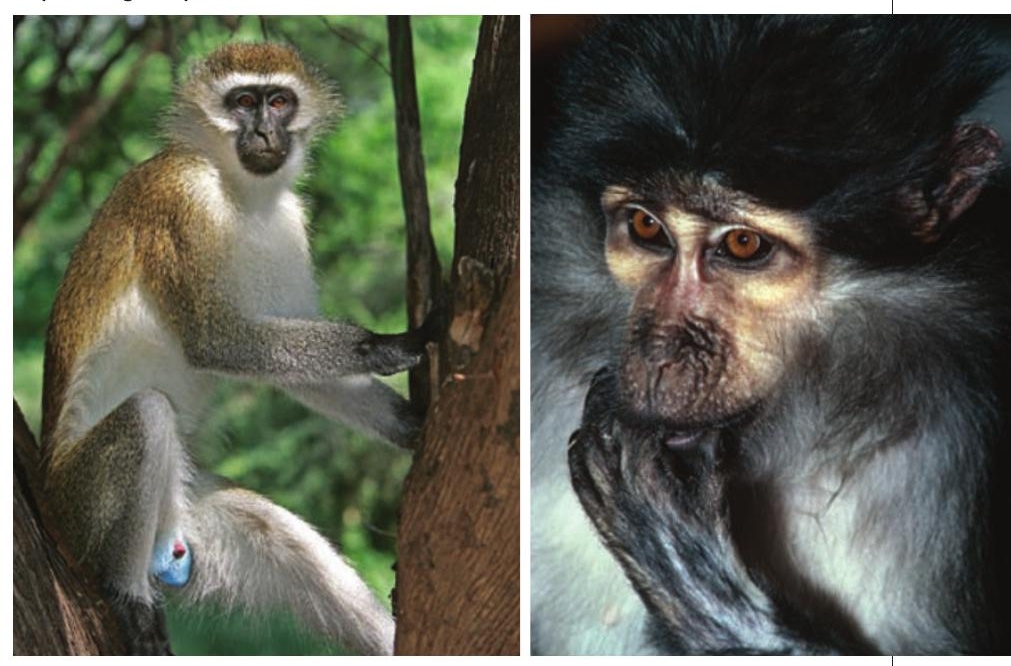

African green monkeys (left) and sooty mangabeys (right) are natural hosts of the HIV-like simian immunodeficiency virus, meaning that they never develop anything resembling AIDS.

and macaques could be the loss of T-helper 17 $\left(\mathrm{T}_{\mathrm{H}} 17\right)$ cells - a subset of CD4 cells that is key for antibacterial defence. Douek and others have shown that SIV-infected sooty mangabeys and African green monkeys have healthy levels of $\mathrm{T}_{\mathrm{H}} 17$ cells in the mucus lining of the gastrointestinal tract. Loss of these cells is a hallmark of progressing infection in humans and macaques (see page S2).

\section{Model monkeys}

In their search for a surrogate for HIV in humans, researchers use about 20 different SIV strains and modified SIVs, dubbed SHIVs, to infect different species of macaques. Each virus-host combination has strengths and limitations.

The main problem with the SIV-macaque model, says Paul Bieniasz, a virologist at the Aaron Diamond AIDS Research Center, New York, is that the SIV genome differs by about $50 \%$ from HIV-1, which is the most prevalent strain worldwide.

To create a better model for vaccine tests, Bieniasz and colleagues are modifying HIV-1 to grow in monkeys. So far, they have created a version that will infect pigtail macaques, initially causing virus levels to reach up to one million copies per millilitre of blood - just like in the initial stages of human infection.

These monkeys do not become sick and, after six months, their infection mimics that seen in long-term non-progressors, who naturally control HIV levels and slow their progression to AIDS (see page S4).

"We basically have to find a way to make the virus more robust in pig-tail macaques," says Bieniasz. "We need it to trigger AIDS."

Bijal Trivedi is a freelance writer in Washington

\section{DC.}

